\title{
Topology optimization for design-dependent hydrostatic pressure loading via the level-set method
}

\author{
Renato Picelli ${ }^{1,2}$ (D) A. Neofytou ${ }^{2} \cdot$ H. Alicia $\mathrm{Kim}^{2,3}$
}

Received: 14 January 2019 / Revised: 22 April 2019 / Accepted: 5 June 2019 / Published online: 19 July 2019 (C) The Author(s) 2019

\begin{abstract}
A few level-set topology optimization (LSTO) methods have been proposed to address complex fluid-structure interaction. Most of them did not explore benchmark fluid pressure loading problems and some of their solutions are inconsistent with those obtained via density-based and binary topology optimization methods. This paper presents a LSTO strategy for designdependent pressure. It employs a fluid field governed by Laplace's equation to compute hydrostatic fluid pressure fields that are loading linear elastic structures. Compliance minimization of these structures is carried out considering the designdependency of the pressure load with moving boundaries. The Ersatz material approach with fixed grid is applied together with work equivalent load integration. Shape sensitivities are used. Numerical results show smooth convergence and good agreement with the solutions obtained by other topology optimization methods.
\end{abstract}

Keywords Topology optimization $\cdot$ Level-set method $\cdot$ Design-dependent load $\cdot$ Fluid pressure $\cdot$ Hydrostatics

\section{Introduction}

The applications of topology optimization are very broad, i.e., multifunctional materials (Guest and Prévost 2006), aeroelasticity (Townsend et al. 2018), biomedical design (Sutradhar et al. 2010), heat transfer (Alexandersen et al. 2016), and acoustics (Azevedo et al. 2018), and others (Bendsøe 1995; Chakraborty et al. 2019). Deaton and Grandhi (2014) identified design-dependent physics as

Responsible Editor: Somanath Nagendra

Renato Picelli

rpicelli@usp.br

$\triangle$ H. Alicia Kim

alicia@ucsd.edu

A. Neofytou

NeofytouA@ cardiff.ac.uk

1 Department of Mining and Petroleum Engineering, University of São Paulo, Praça Narciso de Andrade, Vila Mathias, Santos SP, 11013-560, Brazil

2 Cardiff School of Engineering, Cardiff University, The Queen's Buildings, 14-17 The Parade, Cardiff, CF24 3AA, UK

3 Structural Engineering Department, University of California, San Diego, 9500 Gilman Drive, San Diego, CA 92093, USA one of the eight challenges yet to be addressed within the structural and multidisciplinary optimization field. This class of problems considers different physics (i.e., fluids, heat transfer, electromagnetism, etc.) interacting with structural boundaries and volume changing during optimization.

Design-dependent physics problems can be classified by the type of loads considered, for instance, volumetric or surface loads. The first refers to problems where the loads depend on the volume the body occupies in the medium, e.g., thermal expansion, self-weight loads (Deaton and Grandhi 2016; Huang and Xie 2011). The second type of design-dependent loads acts on the boundaries or surfaces of the body. These can include distributed pressure loads, convective heat transfer, acoustics, and fluidstructure interaction. The main challenge is how to identify, track, and model the multiphysics interface changes during optimization. This paper is focused on problems with design-dependent hydrostatic pressure loads. Such loads are manifested in pump housings, pressure containers, dams, underwater structures, wind loaded structures, and others.

Hammer and Olhoff (2000) pioneered topology optimization for design-dependent pressure loads by developing the parameterization of a load carrying surface within the grayscale density distribution of the solid isotropic material with penalization (SIMP) method. Different parameterization schemes have been proposed over the years ( $\mathrm{Du}$ and 
Olhoff 2004; Zhang et al. 2008; Lee and Martins 2012; Wang et al. 2016). Another way of tracking pressure load surfaces within the SIMP framework uses fluids or multiphysics models. Chen and Kikuchi (2001) and Bourdin and Chambolle (2003) applied a "fluid flooding" technique to systematically identify the pressure surfaces. Sigmund and Clausen (2007) proposed a mixed displacement-pressure finite element (FE) formulation where an overlapping pressure variable is used to define the void phase as a hydrostatic incompressible fluid. Yoon et al. (2007), Yoon (2010), and Lundgaard et al. (2018) extended the idea of using mixed FEs to minimize the dynamic response of acoustic and fluidstructure systems, the latest with the aid of black and white filters (Sigmund 2007).

Motivated by the numerical instabilities that overlapping domains and mixed FE can induce (Brezzi and Fortin 1991), modeling of design-dependent physics with separate domains and classic FE governing equations became another paradigm. The sequence by Picelli et al. (2015a, b, 2017a, b) Vicente et al. (2015) extended the bi-directional evolutionary structural optimization (BESO) method to address design-dependent hydrostatic pressure, acoustic-, and fluid-structure interaction problems with binary $\{0,1\}$ design variables by completely switching fluid and solid elements. Recently, Sivapuram and Picelli (2018) created the topology optimization of binary structures (TOBS) method that benefits from $\{0,1\}$ variables and formal mathematical programming, including the solution for a design-dependent fluid pressure problem. While the SIMP method lacks of explicit fluid-structure interfaces, the BESO and TOBS methods have jaggered boundaries.

The level-set topology optimization (LSTO) method offers well-defined structural boundaries. This is potentially advantageous when handling problems where multiphysics interactions happen at the surface. Shu et al. (2014), Isakari et al. (2017), and Noguchi et al. (2017) proposed different coupling conditions modeling for acoustic-structure problems with the LSTO. In a fluid-structure problem, Jenkins and Maute (2016) combined a level-set method with the extended finite element method (XFEM) to track the deformation of the fluid-structure interfaces and their change during optimization. While a few methodologies have been proposed for design-dependent physics with level set functions in the recent years, the most effective way of modeling pressure loads is still uncertain. It is also not clear how many of these methods would apply to a purely hydrostatic loading problem.

This paper applies a fixed grid FE mesh with the Ersatz material interpolation and, to the best of the authors' knowledge, it is the first one to explore purely hydrostatic pressure loading problems using a discretized fluid domain. The identification of the pressure surfaces is directly obtained via solid-fluid interfaces and their tracking during optimization is done with fluid flooding. Pressure loads are applied on the structure with work equivalent integrals. The work equivalent loads were first used in a level-set method with the Ersatz material interpolation by Emmendoerfer et al. (2018) and they are employed here due to its simplicity. Emmendoerfer et al. (2018) also used fluid flooding, but no actual fluid pressure is solved in their work. The addition of a fluid pressure field governed by Poisson's equation and the use of shape sensitivities are explored in this paper, as well as the application of the LSTO method previously used to address stress-based problems Picelli et al. (2018a, b). We apply the LSTO method to the challenging pressure chamber example originally proposed by Hammer and Olhoff (2000), which, to the authors' best knowledge, has not been attempted with LSTO in the literature.

The remainder of the paper is organized as follows. Section 2 describes the governing equations, finite element method, and the formulation for tracking the fluid-structure interface by fluid flooding. In Section 3, the levelset topology optimization method is outlined. Section 4 presents and discusses numerical results, and Section 5 concludes the paper.

\section{Governing equations and finite element model}

We apply the static analysis of elastic structures in contact with incompressible hydrostatic fluids. The solid domain is considered to be under small strain condition and the fluid domain is inviscid and irrotational. This is a partially coupled problem and, in this work, both domains are solved separately. First, the fluid pressure field is obtained. With the fluid solution and the identification of the fluid-structure interface, the structural analysis is carried out assembling the distributed pressure loads. The bilinear elements and isoparametric mapping are used for both domains.

\subsection{Fluid analysis}

Consider a hydrostatic fluid domain $\Omega_{f}$ illustrated in Fig. 1 governed by Laplace's equation

$\nabla^{2} P_{f}=0 \quad$ in $\Omega_{f}$,

where $P_{f}$ is the fluid pressure and $\nabla^{2}$ is the Laplacian differential operator. Boundary conditions include an imposed pressure $P_{0}$ on a portion $\Gamma_{p}$ of the fluid boundary as

$P_{f}=P_{0} \quad$ on $\Gamma_{p}$, 
and the hard wall condition

$\mathbf{n} \cdot \nabla P_{f}=0 \quad$ on $\Gamma_{w}$,

where $\mathbf{n}$ is the outward unit normal vector to the fluid and $\nabla$ is the gradient vector operator, imposed on the portion $\Gamma_{w}$ of the total fluid boundary $\Gamma_{f}=\Gamma_{f} \cup \Gamma_{w}$. The discretization of (1) via the finite element method (FEM) yields

$\mathbf{K}_{f} \mathbf{P}_{f}=\mathbf{f}_{f}$,

where $\mathbf{P}_{f}$ is the vector of pressures and $\mathbf{f}_{f}$ is the equivalent load to enforce the Dirichlet boundary condition from (2) computed, for instance, using a penalty method (Bathe 2006). The global fluid stiffness matrix $\mathbf{K}_{f}$ is computed as

$\mathbf{K}_{f}=\mathrm{A}_{e=1}^{n e f} \int_{\Omega_{f e}}\left(\nabla \mathbf{N}_{f}\right)^{T} \nabla \mathbf{N}_{f} d \Omega_{f e}$,

where $n e f$ is the total number of fluid elements and $\mathbf{N}_{f}$ is the vector with shape functions of the fluid element $\Omega_{f e}$. The solution of (4) provides the pressure field used in the following structural analysis.

\subsection{Structural analysis}

The considered structural domain $\Omega_{s}$ shown in Fig. 1 bounded by $\Gamma_{s}=\Gamma_{d} \cup \Gamma_{n} \cup \Gamma_{h}$ is governed by the linear elasticity equation expressed, disregarding body loads, as

$\nabla \cdot \boldsymbol{\sigma}_{s}\left(\mathbf{u}_{s}\right)=\mathbf{f}_{s} \quad$ on $\Omega_{s}$,

where $\sigma_{s}\left(\mathbf{u}_{s}\right)$ is the Cauchy stress tensor and $\mathbf{u}_{s}$ is the structural displacement field. The right-hand side of (6) includes the loads $\mathbf{f}_{s}$ applied on the structure. Dirichlet boundary conditions are applied on the portion $\Gamma_{d}$ of the structural boundary as

$\mathbf{u}_{s}=\mathbf{u}_{0} \quad$ on $\Gamma_{d}$,

where $\mathbf{u}_{0}$ is the vector of constrained displacements. Neumann boundary conditions are applied by assembling $\mathbf{f}_{p}$ on the boundary portion $\Gamma_{n}$ of the structure. The boundary $\Gamma_{h}$ is the portion with no boundary conditions and only the subset $\Gamma_{n} \cup \Gamma_{h}$ is subjected to optimization. A void domain

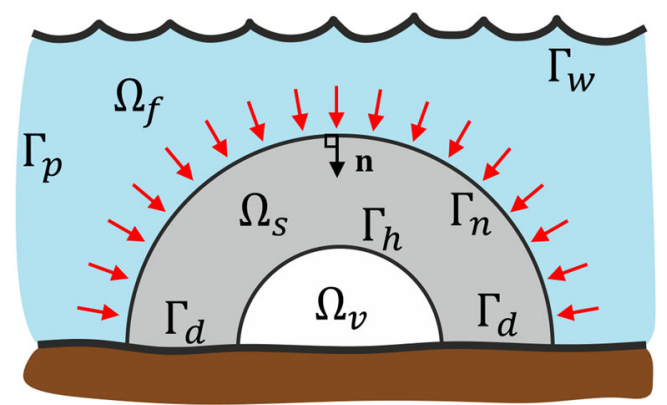

Fig. 1 Representation of a structure under hydrostatic loads
$\Omega_{v}$ represents holes inside the design region. In the FEM discretization, (6) becomes

$\mathbf{K}_{s} \tilde{\mathbf{u}}_{s}=\mathbf{f}_{p}$,

where $\tilde{\mathbf{u}}_{s}$ is the vector of structural displacements and $\mathbf{K}_{s}$ is the structural stiffness matrix computed as

$\mathbf{K}_{s}=\mathrm{A}_{e=1}^{\text {nes }} \int_{\Omega_{s e}} \mathbf{B}^{T} \mathbf{D B} \Omega_{s e}$,

with $\mathbf{B}$ being the strain-displacement matrix, $\mathbf{D}$ the constitutive matrix, nes the total number of finite structural elements in the mesh, and $\Omega_{s e}$ indicates the element domain. In this work, the applied loads are only in the form of distributed pressure loads, represented by $\mathbf{f}_{p}$. These governing equations are general and describe the fluid and structural analyses for 2D and 3D.

In the fixed grid level-set method, the structural boundaries intersect the elements and the pressure load $\mathbf{f}_{p}$ cannot be directly applied at those boundaries. As illustrated in 2D in Fig. 2, work equivalent nodal forces $\mathbf{f}_{e}$ can be computed by integrating along the cutting boundary the matrix product of the element shape functions $\mathbf{N}_{s}$ and the distributed pressure loading acting on the element as

$\mathbf{f}_{p}=\bigwedge_{k=1}^{n s} \mathbf{f}_{k}$,

with $n s$ being the total number pressure load segments and

$\mathbf{f}_{k}=\int_{L_{k}} \mathbf{N}_{s}^{T} \mathbf{f}_{p k} d L_{k} \quad$ on $\Gamma_{n}$,

where $L_{k}$ is the $k$ th segment defined by the cutting boundary $\Gamma_{n}$ and $\mathbf{f}_{p k}$ is the pressure loading acting on that element (Cook et al. 2002).

The integral from (11) can be computed via Gaussian quadrature; therefore, it can then be rewritten as

$\mathbf{f}_{k}=\int_{-1}^{1} \mathbf{N}_{s}\left(\mathbf{g}_{\mathrm{mod}}\right)^{T} \mathbf{f}_{p k} d \xi$,

where the integral interval over the line $L_{k}$ is changed to $[-1,1]$ and the Gaussian points are modified to reflect the element local coordinates, herein represented by $\mathbf{g}_{\text {mod }}$. We have implemented the quadrature using one Gauss point, i.e., the midpoint of the line $L$. For this, the modified Gauss point coordinates are

$\mathbf{g}_{\text {mod }}=\left\{\begin{array}{l}\left(\mathrm{x}_{g}-\mathrm{x}_{c}\right) / a \\ \left(\mathrm{y}_{g}-\mathrm{y}_{c}\right) / b\end{array}\right\}$,

where $\left\{\mathrm{x}_{g}, \mathrm{y}_{g}\right\}$ and $\left\{\mathrm{x}_{c}, \mathrm{y}_{c}\right\}$ are the global coordinates of the Gauss point and the element centroid, respectively. The terms $a$ and $b$ are, respectively, half of length and height of 
Fig. 2 Work equivalent nodal loads computed with an integral over the segment $L_{k}$

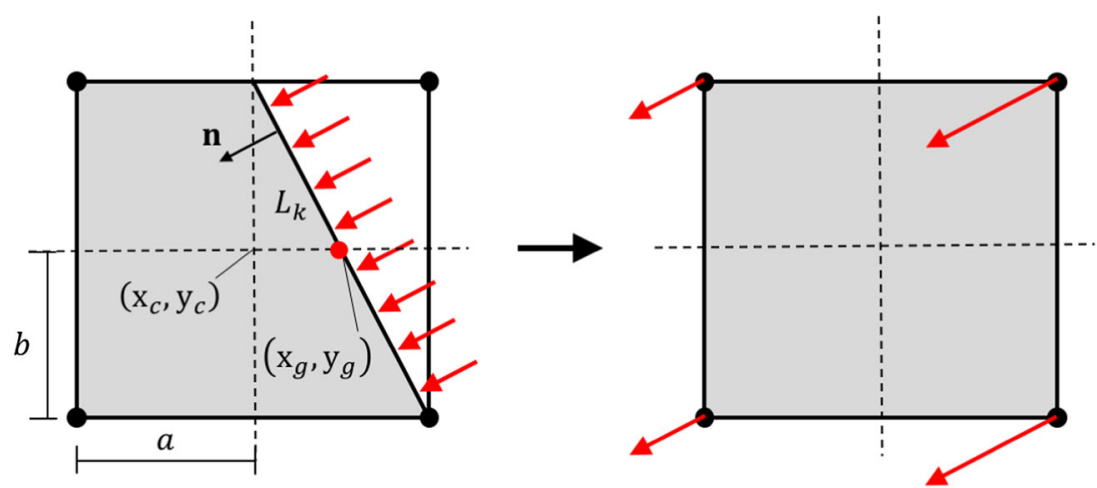

the element (see Fig. 11). Considering that the distributed pressure is expressed by

$\mathbf{f}_{p k}=P_{f} \mathbf{n}$,

Equation (12) is rewritten as

$\mathbf{f}_{k}=P_{f} \mathbf{N}_{s}\left(\mathbf{g}_{\mathrm{mod}}\right)^{T} \mathbf{n} w J$,

where,

$\mathbf{N}_{s}\left(\mathbf{g}_{\text {mod }}\right)=\left[\begin{array}{ccccc}N_{1}\left(\mathbf{g}_{\text {mod }}\right) & 0 & \ldots & N_{4}\left(\mathbf{g}_{\text {mod }}\right) & 0 \\ 0 & N_{1}\left(\mathbf{g}_{\text {mod }}\right) & \ldots & 0 & N_{4}\left(\mathbf{g}_{\text {mod }}\right)\end{array}\right]$,

$w=2$ being the weight of the Gaussian quadrature for one point and $J$ the Jacobian given, in this case, as half of the length of $L_{k}$. This classic FE procedure is a much simpler alternative to XFEM and mixed models.

\subsection{Interface tracking}

Fluid flooding as first called by Chen and Kikuchi (2001) is used to track the fluid-structure interfaces. The process consists in defining an initial fixed fluid region that is propagated to the adjacent neighbor elements or nodes until it finds a structural boundary. In the FEM framework, this can be done by creating a list of element types, verifying

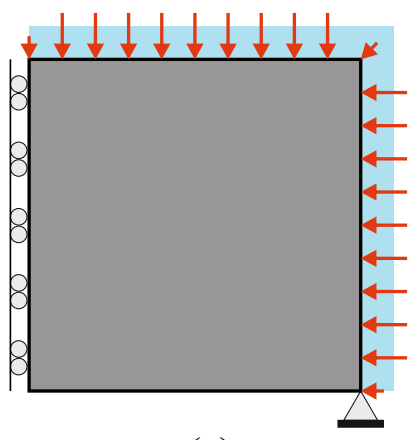

(a)

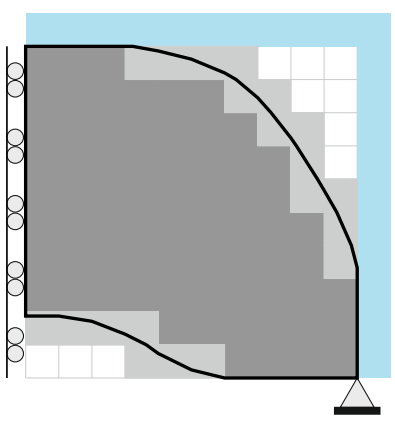

(b)

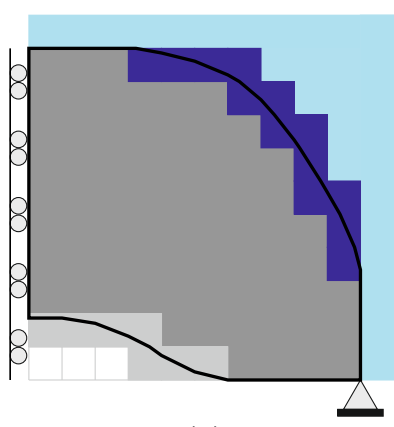

(c)

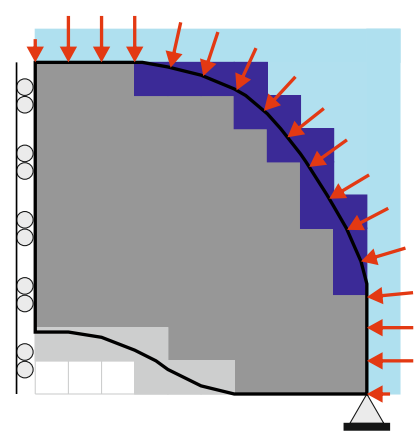

(d)

Fig. 3 Interface tracking procedure via fluid flooding. a Fluid-structure design with pressure load assembly. b New fluid-structure design. c Fluid flooding process. $\mathbf{d}$ New pressure load assembly 
defined as

$\left\{\begin{array}{l}\phi(\mathrm{x}) \geq 0, \mathrm{x} \in \Omega_{s}, \\ \phi(\mathrm{x})=0, \mathrm{x} \in \Gamma_{s}, \\ \phi(\mathrm{x})<0, \mathrm{x} \notin \Omega_{s},\end{array}\right.$

where $\mathrm{x}$ is any point inside the design region $\Omega_{d}$ that contains the structural domain $\Omega_{s}$. The boundary of the structure can be changed implicitly through $\phi(\mathrm{x})$. This way of representing the structure allows its boundary to morph by solving the following Hamilton-Jacobi equation,

$\frac{d \phi(\mathrm{x}, t)}{d t}+|\nabla \phi(\mathrm{x})| V_{n}(\mathrm{x})=0$

where $V_{n}$ is the normal velocity and $t$ is a pseudo time domain in which the level set evolves.

Equation (18) is discretized and solved numerically as

$\phi_{i}(t+\Delta t)=\phi_{i}(t)-\Delta t\left|\nabla \phi_{i}(t)\right| V_{n}^{i}(t)$,

where $i$ is a discrete point in the domain and $\left|\nabla \phi_{i}(t)\right|$ is computed using the Hamilton-Jacobi WENO (weighted essentially non-oscillatory) scheme (Osher and Fedkiw 2003). The update of (19) it is conveniently restricted to nodes within a narrow band close to the boundary. Periodically, the level-set function $\phi_{i}$ is reinitialized to a signed distance function in order to maintain the stability of the method. The reinitialization and velocity extension are done via the fast-marching method (Sethian 1996).

\subsection{Linearized sub-problem}

The velocity function required to change the implicit function $\phi_{j}$ in (19) can be obtained by solving the following linearized problem,

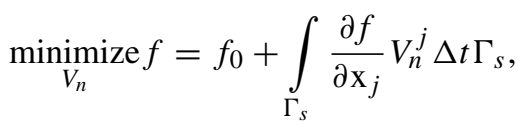

subject tog $g^{m}=g_{0}^{m}+\int_{\Gamma_{s}} \frac{\partial g^{m}}{\partial \mathrm{x}_{j}} V_{n}^{j} \Delta t \Gamma_{s} \leq \bar{g}^{m}$,

$$
\left|V_{n}^{j} \Delta t\right| \leq d_{\mathrm{cfl}}
$$

where $f$ is the objective function, $g^{m}$ is the $m$ th constraint with constrained value $\bar{g}^{m}, V_{n}^{j} \Delta t=\Delta \mathrm{x}_{j}$, and $d_{\mathrm{cfl}}$ is the maximum distance the boundary point can move along the normal direction inside the design region, i.e., the CourantFriedrichs-Lewy (CFL) stability condition.

The integrals in (20) can be estimated as,

$$
\int_{\Gamma_{s}} \frac{\partial f}{\partial \mathrm{x}_{j}} V_{n}^{j} \Delta t \Gamma_{s} \approx \sum_{j=1}^{n b} l_{j} \frac{\partial f}{\partial \mathrm{x}_{j}} V_{n}^{j} \Delta t=\mathbf{S}_{f} \cdot \mathbf{V}_{n} \Delta t
$$

and,

$\int_{\Gamma_{s}} \frac{\partial g^{m}}{\partial \mathrm{x}_{j}} V_{n}^{j} \Delta t \Gamma_{s} \approx \sum_{j=1}^{n b} l_{j} \frac{\partial g^{m}}{\partial \mathrm{x}_{j}} V_{n}^{j} \Delta t=\mathbf{S}_{g} \cdot \mathbf{V}_{n} \Delta t$

where $l_{j}$ is the discrete length of the boundary around the boundary point $j, \mathbf{S}_{f}$ and $\mathbf{S}_{g}$ are vectors containing the product of boundary lengths and sensitivities, and $\mathbf{V}_{n}$ is the vector of normal velocities. With (21) and (22), $\mathbf{V}_{n}$ can be found in order to minimize the objective function, given any constraints. This provides a numerical implementation of the steepest-descent of the objective function for the set of $\mathbf{V}_{n}$. However, we switch the problem to a variable time step that is optimized according to the values of the sensitivities as

$\mathbf{V}_{n} \Delta t=-\lambda_{f} \mathbf{S}_{f}+\lambda_{g_{1}} \mathbf{S}_{g^{1}}+\lambda_{g^{2}} \mathbf{S}_{g^{2}}+\ldots+\lambda_{g^{m}} \mathbf{S}_{g^{m}}$,

where $\lambda_{f}$ and $\lambda_{g^{1}}+\lambda_{g^{2}}+\ldots+\lambda_{g^{m}}$ are the optimization variables to be determined. We then reformulate (20) as

$$
\begin{array}{cl}
\underset{\lambda_{f}, \lambda_{g}}{\operatorname{minimize}} & \mathbf{S}_{f} \cdot \mathbf{V}_{n}\left(\lambda_{f}, \lambda_{g^{m}}\right) \Delta t, \\
\text { subject to } & \mathbf{S}_{g} \cdot \mathbf{V}_{n}\left(\lambda_{f}, \lambda_{g^{m}}\right) \Delta t \leq \Delta \bar{g}, \\
& \left|\mathbf{V}_{n} \Delta t\right| \leq \mathbf{d}_{\mathrm{cfl}},
\end{array}
$$

where $\mathbf{d}_{\mathrm{cfl}}$ includes the CFL condition and any other possible bounds for the movement of each boundary point, e.g., to restrict a point from moving out of the design domain. The term $\Delta \bar{g}$ is the allowed change of each constraint function at every iteration.

In summary, the optimization problem from (24) is solved for $\lambda_{f}$ and $\lambda_{g^{m}}$, therefore considerably reducing the number of optimization variables from the number of boundary points to the number of objective and constraint functions. For instance, when doing compliance minimization subject to volume constraints, the amount of optimization variables is only two, independently of the boundary size. This procedure enforces the velocity field to be a linear combination of the sensitivity fields, leading to a smooth propagation of the structural boundaries. This is also advantageous when handling a high number of constraints, as shown in Dunning et al. (2016). More details of this optimization formulation can be found in Picelli et al. (2018a) and Hedges et al. (2017).

\subsection{Optimization problem and shape sensitivities}

Compliance minimization of structures under designdependent pressure loads subject to a volume constraint is considered. This optimization problem, $P_{1}$, can be written as

$$
\begin{array}{lll} 
& \text { minimize } & \mathbf{f}_{p}^{T} \tilde{\mathbf{u}}_{s}, \\
P_{1} \quad \text { subject to } & \mathbf{K}_{s} \tilde{\mathbf{u}}_{s}=\mathbf{f}_{p}, \\
& V_{s} \leq \bar{V},
\end{array}
$$




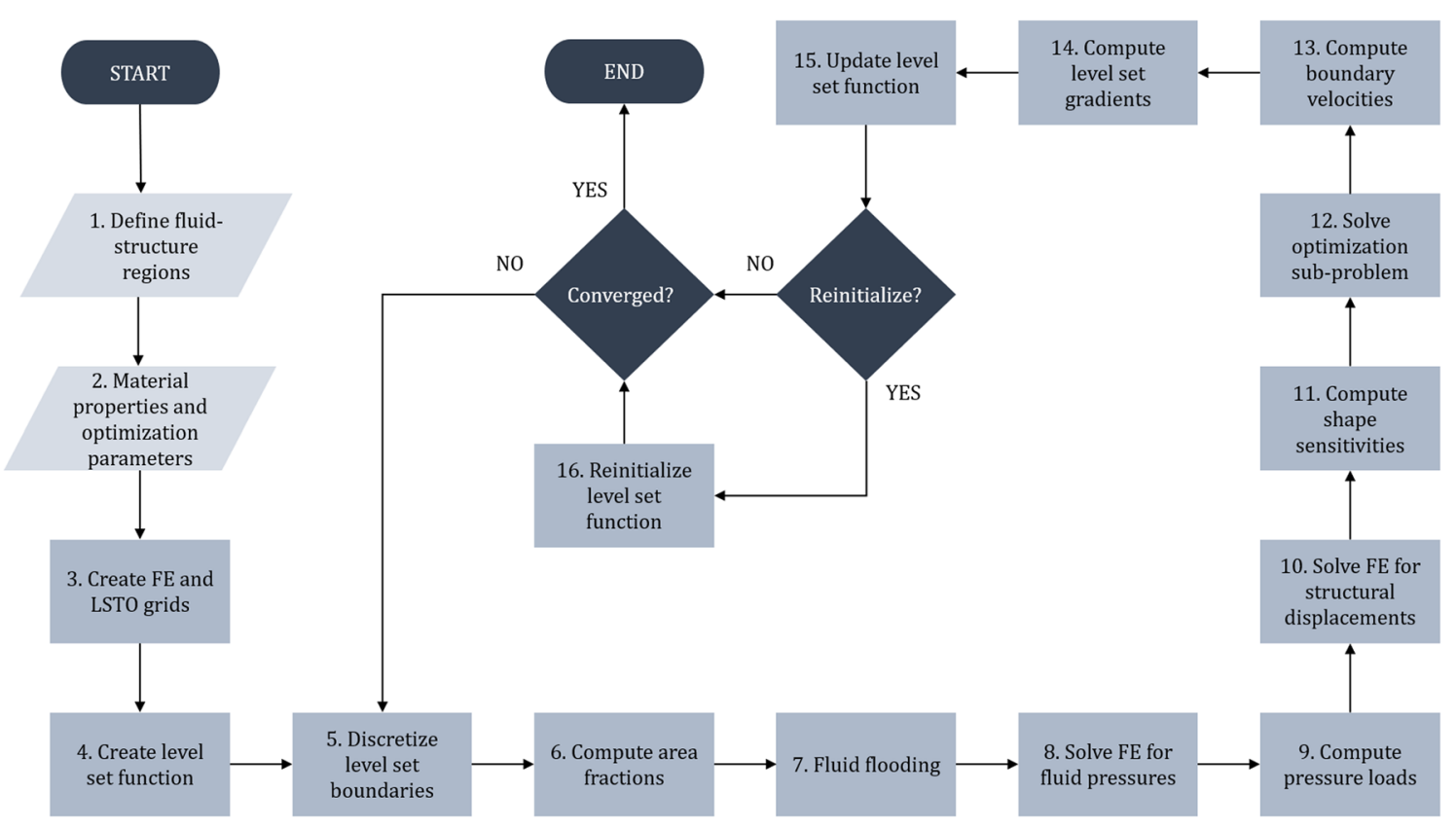

Fig. 4 Flowchart of the optimization procedure

where $V_{S}$ is the volume fraction that the structure occupies regarding the design domain and $\bar{V}$ is the maximum allowed volume fraction.

For the LSTO method, regularization techniques (e.g., length scale and perimeter control) have been often used to reduce the number of local minima and make the problem well-posed. We acknowledge that the use of perimeter control, for instance, mitigates the dependency on the mesh size and on the initial level-set function of the problem, i.e., the initial hole configuration. We, therefore, also formulate the following optimization problem, $P_{2}$, including a perimeter term in the objective. The needs and effects of the perimeter regularization term will further be discussed in Section 4.

$$
\begin{array}{ccl} 
& \text { minimize } & \mathbf{f}_{p}^{T} \tilde{\mathbf{u}}_{s}+\mu \chi_{s}, \\
P_{2} \quad \text { subject to } & \mathbf{K}_{s} \tilde{\mathbf{u}}_{s}=\mathbf{f}_{p}, \\
& V_{s} \leq \bar{V},
\end{array}
$$

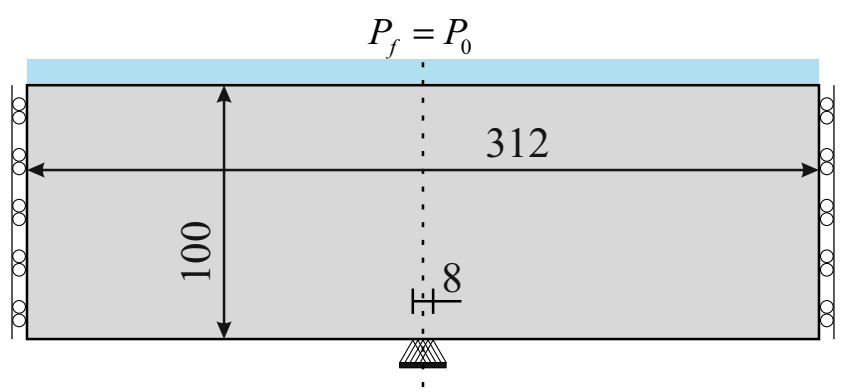

Fig. 5 Piston problem scheme considered for optimization where $\mu$ is a fixed positive parameter and $\chi_{s}$ is the perimeter of the structure given by

$\chi_{s}=\int_{\Gamma_{s}} d \Gamma_{s}$.

This work applies shape sensitivities to morph the structural boundaries towards an optimal solution. Shape sensitivities for a structural compliance function considering design-dependent pressure loads have been studied in Allaire et al. (2004) and Xia et al. (2015). Including the perimeter parameter, the shape sensitivity can be written as,

$\frac{\partial f}{\partial \mathrm{x}}=\int_{\Gamma_{s}}\left[2 \operatorname{div}\left(p_{0} u\right)-D \varepsilon(u) \cdot \varepsilon(u)+\mu \kappa\right] V_{n} d \Gamma_{s}$,

where $p_{0}$ is the pressure load, $u$ is the displacement, $D \varepsilon(u)$ is the stress tensor, $\varepsilon(u)$ is the strain tensor, and $\kappa$ is the curvature of the boundary.

Equation (28) represents the shape sensitivity in the continuum space for the objective in $P_{2}$. One advantage of employing shape sensitivities is that it allows the use

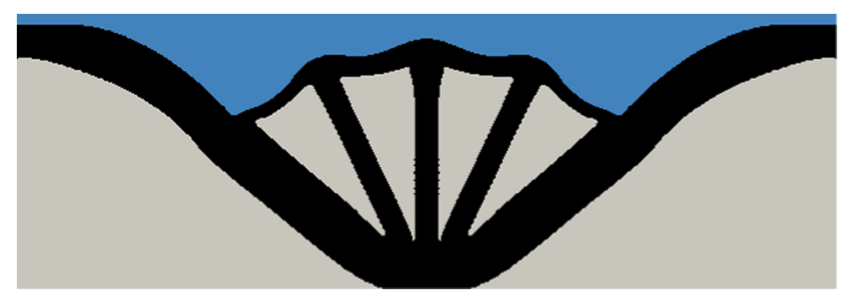

Fig. 6 Piston design solution 
Fig. 7 Snapshots of the piston design via LSTO

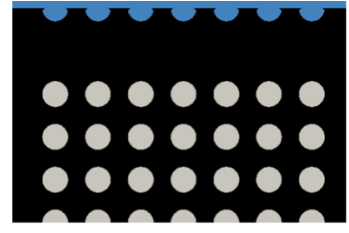

It.: 0

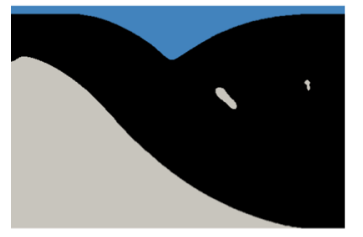

It.: 75

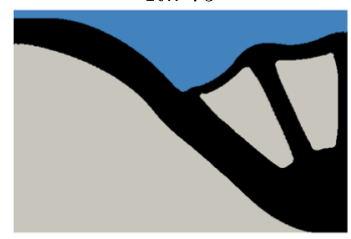

It.: 200

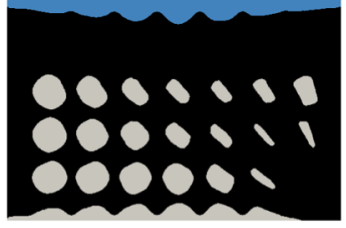

It.: 25

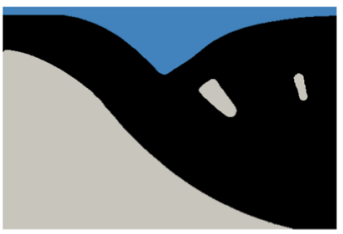

It.: 100

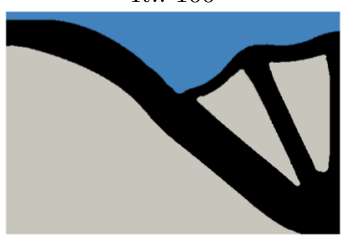

It.: 250

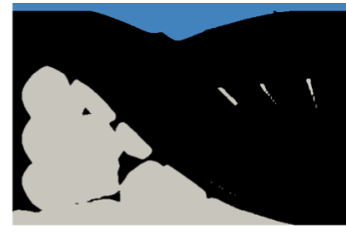

It.: 50

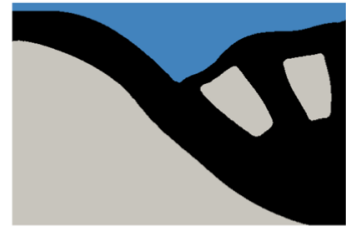

It.: 150

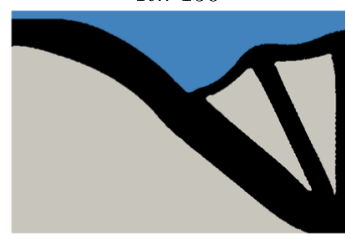

It.: 333 of any analysis method (FEM or others) to compute the terms in sensitivity function, possibly allowing it to be directly associated with black-box commercial packages. In this case, (28) indicates that the sensitivity of a boundary point depends on the divergence of the pressure times displacement, stress times strain, and lastly, the curvature of the boundary. In this work, the FEM is employed to compute (28) as

$\frac{\partial f}{\partial \mathbf{x}_{j}}=\left[2\left(P_{f} \mathbf{B} \tilde{\mathbf{u}}_{s} \cdot\{1,1,0\}+\tilde{\mathbf{u}}_{s} \cdot \nabla \mathbf{N}_{f} P_{f}\right)-\mathbf{D B} \tilde{\mathbf{u}}_{s} \cdot \mathbf{B} \tilde{\mathbf{u}}_{s}+\mu \kappa\right]^{j}$.

In the fixed grid LSTO, the stiffness of the elements cut by the structural boundaries is weighted by the area fraction of those elements. This is popularly known as the Ersatz material interpolation. Another advantage of the present LSTO method is that a least-squares interpolation is used to recover the continuity of the structural response at the boundary points. This allows a fixed grid to be used with desired accuracy and showed to produce smooth topologies in our stress-based optimization work (Picelli et al. 2018a). This procedure is used here. First, the terms in (29) that depend on displacements are computed at the Gauss points of the elements, where the FEM gives high accuracy when the element is not distorted (Zienkiewicz and Taylor 2005). Each term is then interpolated via least squares at the boundary point $\mathrm{x}_{j}$. For the free boundaries $\Gamma_{h}$, the terms depending on $P_{f}$ in (28) and (29) are null. For $P_{1}$, in which no perimeter function is considered, the term $\mu \kappa$ is zero. For $P_{2}$, this term is computed via the finite difference method. One can efficiently compute the perimeter sensitivities by the local difference in the length of each point segment with a small perturbation of each boundary point coordinate in the normal direction. The least squares and perimeter sensitivities can be computed using our open source code available at http://m2do.ucsd.edu/software/.

\subsection{Method summary}

Figure 4 provides the flowchart of the present optimization procedure. The list of steps is as follows:

1. Define the region where the structure occupies, a structural design domain and a fixed fluid region.

2. Choose material properties and optimization parameters.

3. Instantiate the finite element mesh and the level-set grid. One finite element mesh is able to cover both fluid and solid domains.

4. Initialize the level-set function $\phi_{i}$ for an initial structural design, e.g., including initial holes.

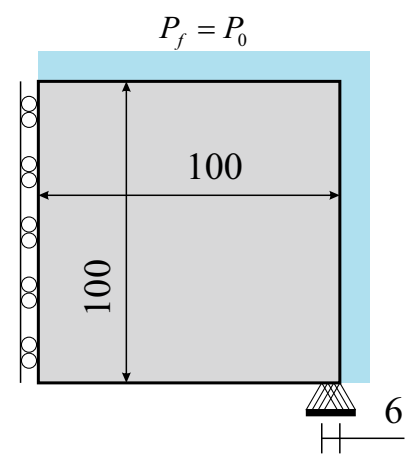

Fig. 8 Design example considered for optimization to obtain an arch structure solution 
Fig. 9 Arch structure solutions for a $P_{1}$ with final compliance $7.74 \times 10^{4}$ and $\mathbf{b} P_{2}$ with final compliance $7.39 \times 10^{4}$

Fig. 10 Snapshots of the arch structure design via LSTO

Fig. 11 a Pressurized chamber example considered for optimization and $\mathbf{b}$ initial guess design

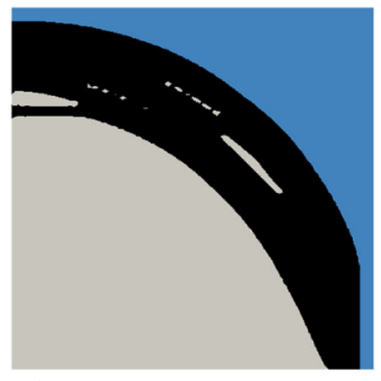

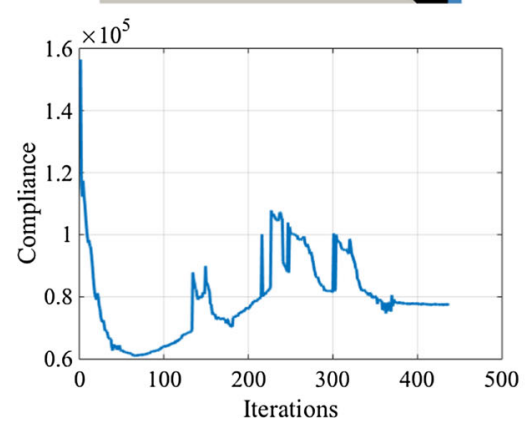

(a)
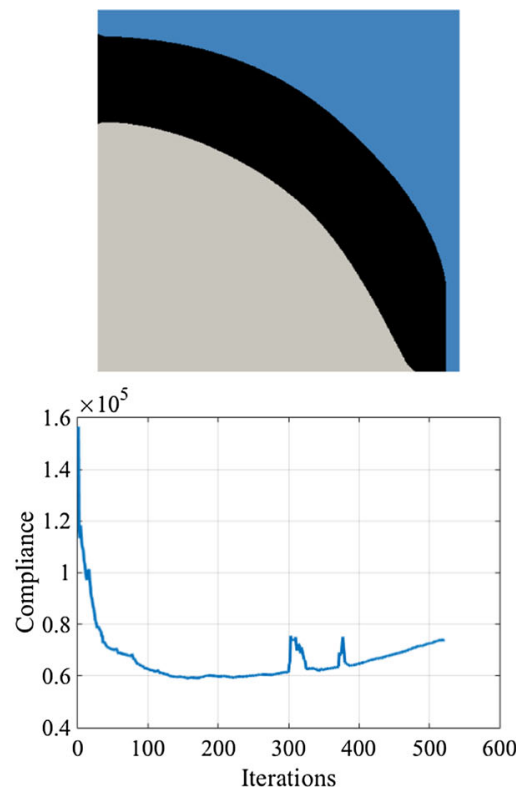

(b)

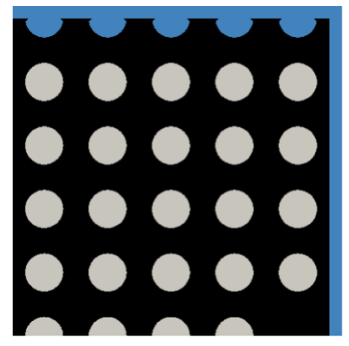

It.: 0

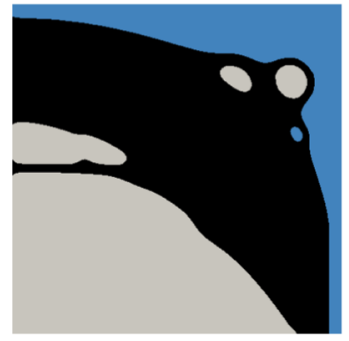

It.: 300

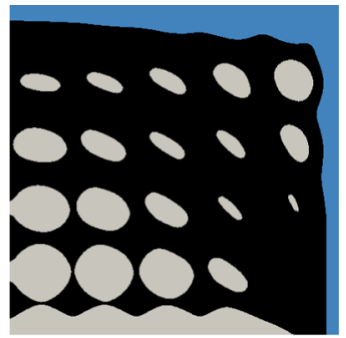

It.: 100

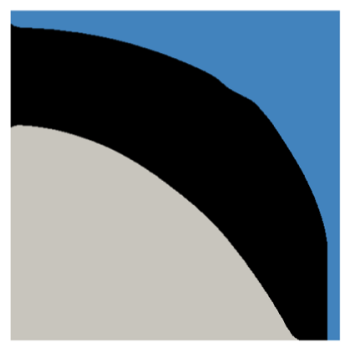

It.: 400

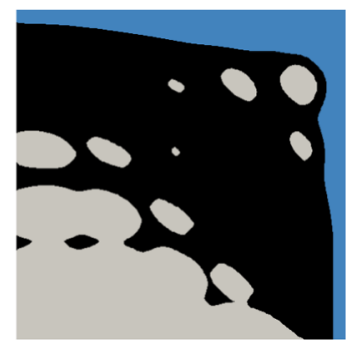

It.: 200

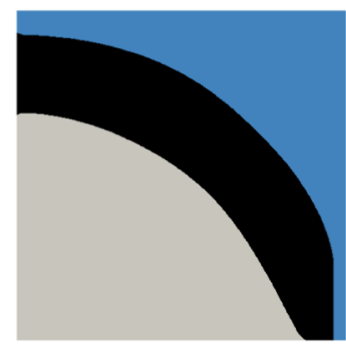

It.: 523

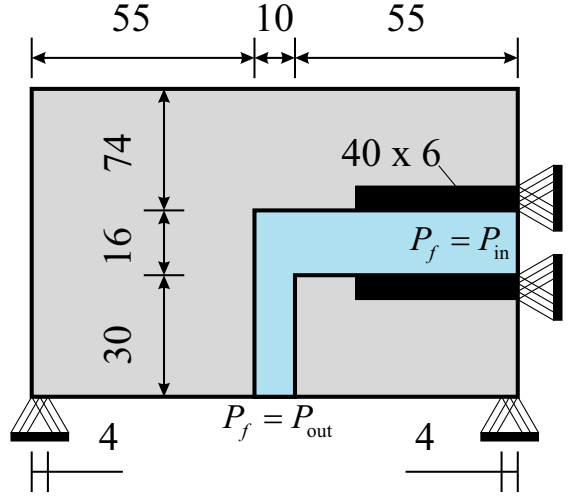

(a)

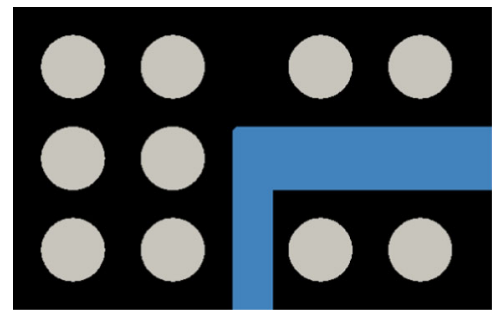

(b) 
Fig. 12 Chambers solutions for different $P_{\text {in }}$ 's

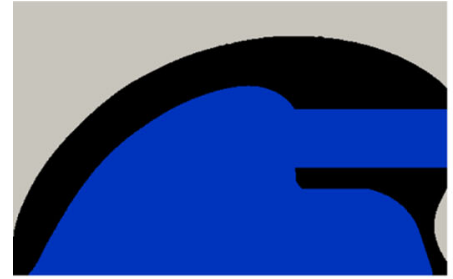

$P_{\text {in }}=1.0$

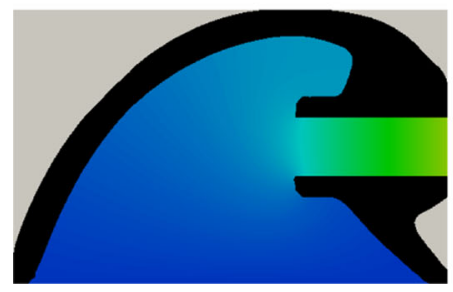

$P_{\text {in }}=10.0$

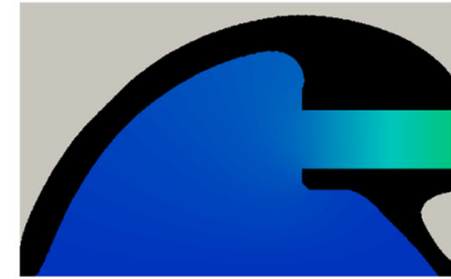

$P_{\text {in }}=5.0$

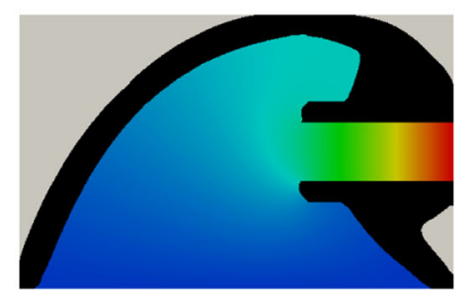

$P_{\text {in }}=15.0$
Pressure

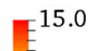

$-12.0$

$=9.00$

$=6.00$

$=3.00$

0.00
5. Discretize the level-set boundaries into a set of boundary points and segments.

6. Compute the area fractions for each element.

7. Propagate the fluid element status according to the procedure described in Section 2.3 and identify the segments carrying pressure loads.

8. Assemble and solve the finite element model from (4) to obtain the fluid pressure field.

9. Compute and assemble the pressure loads for the structural analysis with (10).

10. Assemble the stiffness matrix and solve (8) to obtain the displacements field $\tilde{\mathbf{u}}_{s}$.

11. Compute the discretized shape sensitivity function from (29) at all boundary points via least-squares interpolation.

12. Solve the optimization sub-problem from (24) for optimal $\lambda_{f}$ and $\lambda_{g^{m}}$.

13. Compute the velocities at boundary points using (23).

14. Compute the level-set gradients $\left|\nabla \phi_{j}\right|$ at boundary points.

15. Update level-set function $\phi_{i}$ at the grid nodes around the boundaries using (19).

16. Reinitialize level-set function if necessary/desired.

17. Check convergence, e.g., the change in the objective function during the last five iterations.

18. If not converged, return to step 5 and iterate.

\section{Numerical results}

This section explores and discusses the benchmark examples for design-dependent pressure loading problems in topology optimization. A solid material with Young's mod- ulus of 1.0 and Poisson's ratio 0.3 is used in the structural analysis for all examples. Plane stress condition is assumed. The convergence is reached if the change in the structural compliance function during five consecutive iterations is less than 0.001 .

\subsection{Piston design}

The schematic piston design problem from Fig. 5 is considered for optimization. To the best of the authors' knowledge, this problem was first explored by Bourdin and Chambolle (2003) and later used by several other works for a range of different methods (Sigmund and Clausen 2007; Lee and Martins 2012; Picelli et al. 2015b; Emmendoerfer et al. 2018). This schematic problem is explored here to verify the topology solution offered by our proposed method.

The left half of the model in Fig. 5 is solved here with $156 \times 104(156 \times 100$ for the structure $)$ quadrilateral finite elements in a regular fixed mesh. The problem $P_{1}$ from (25) without a regularization is solved for final volume fraction $\bar{V}=30 \%$. Boundary conditions include the symmetry at the center line and an imposed fluid pressure $P_{0}=1.0$ per unit length at the top edge of the fluid domain. Figure 6 presents the mirrored solution of the piston design problem with the proposed LSTO method.

The topology solution from Fig. 6 agrees well with the existing solutions, e.g., by Lee and Martins (2012) and Wang et al. (2016) using the SIMP method and Picelli et al. (2015b) using the BESO method. They have the central holes converging to the bottom center support, convex shapes of the top structural members (resembling seashells), and the curved shapes of the structures touching the lateral 
Fig. 13 Snapshots of the pressurized chamber design via LSTO for $P_{\text {in }}=10.0$

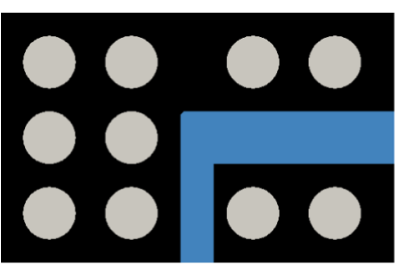

It.: 0

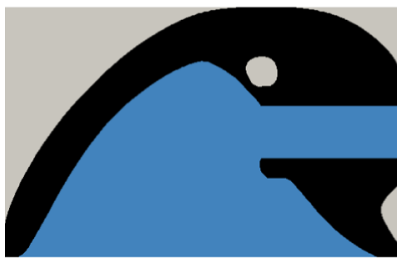

It.: 150

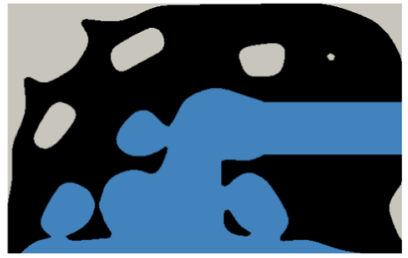

It.: 50

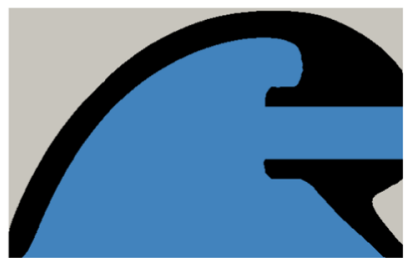

It.: 200

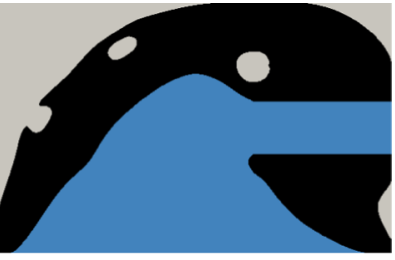

It.: 100

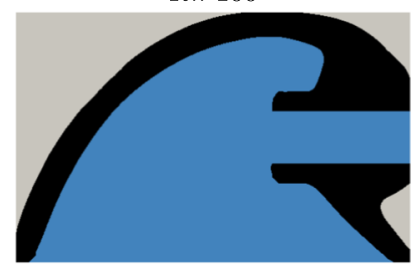

It.: 256 walls. They mainly differ between each other in the amount of internal structural members. The piston design by Sigmund and Clausen (2007) is less similar to the seashell shapes and the solutions are dependent on the control of the fluid volume. Regarding other level-set methods, the solutions presented in the literature like in Emmendoerfer et al. (2018) and Xia et al. (2015) are noticeably different. The overall looks of the piston design from Emmendoerfer et al. (2018) and this work are similar, but the shapes and position of the central and top structural members in Emmendoerfer et al. (2018) are somehow altered. The solution obtained by Xia et al. (2015) is evidently less similar than Emmendoerfer et al. (2018) and this work, presenting different shapes and holes configuration. Bourdin and Chambolle (2003) applied a phase field to represent the fluid-solid-void of the piston design. Their solution is similar to the ones obtained by the other methods only when a perimeter penalization is high enough. When such penalization is lowered, a different set of central structural members are obtained. Figure 7 presents snapshots of our LSTO solution. The initial solution chosen is similar to the one presented by Xia et al. (2015).

\subsection{Arch structure}

The piston design problem demonstrated that the solution of the proposed LSTO method converges to the ones in the literature without regularization. This following example explores the perimeter integral in the objective function and compares problems $P_{1}$ and $P_{2}$ from (25) and (26), respectively. Herein, the model depicted in Fig. 8 is considered for optimization. A fixed grid with $104 \times$ 104 quadrilateral finite elements is used, with the structural design domain of $100 \times 100$. Both problems $P_{1}$ and $P_{2}$ are solved for comparison, with the volume constraint $\bar{V}=$ $30 \%$ and $\mu=1.0$. The fluid pressure $P_{0}=1.0$ per unit length is applied. The solutions are shown in Fig. 9.

Figure 9 a presents the solution without the perimeter regularization, and the solution has small holes in the arch structure. A close investigation of the strain energy and sensitivities revealed that their distributions are near uniform and the volume constraint is satisfied. This means that small holes have little effects in the compliance function. A similar effect was observed in Picelli et al. (2017a). In such cases, these holes remain in the final solution when solving $P_{1}$. Solving problem $P_{2}$ penalizes the perimeter and the small holes do not remain in the final solution as shown in Fig. 9 b. The difference in the compliance function values between these two solutions is $4.6 \%$. Figure 9 a shows the convergence history of the structural compliance function when solving $P_{1}$. Sharp changes are observed due to the fact that several holes are merging and structural members are breaking during

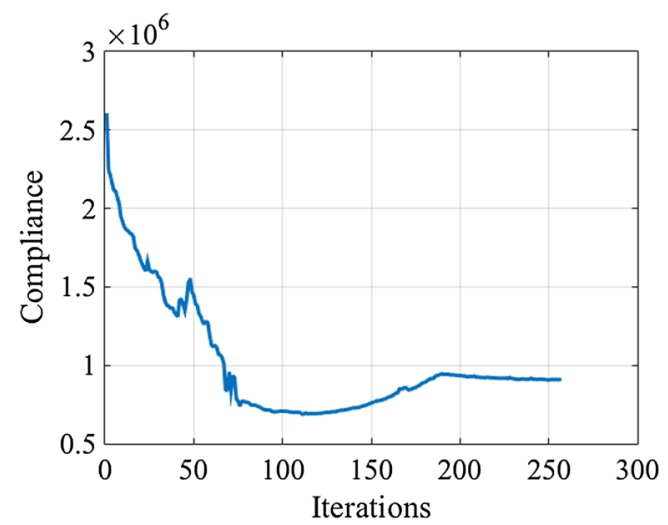

Fig. 14 Objective function history for the chamber design problem 
Fig. 15 Sensitivity function values when $\mathbf{a} P_{\text {in }}=1.0$ and $\mathbf{b}$ $P_{\text {in }}=10.0$

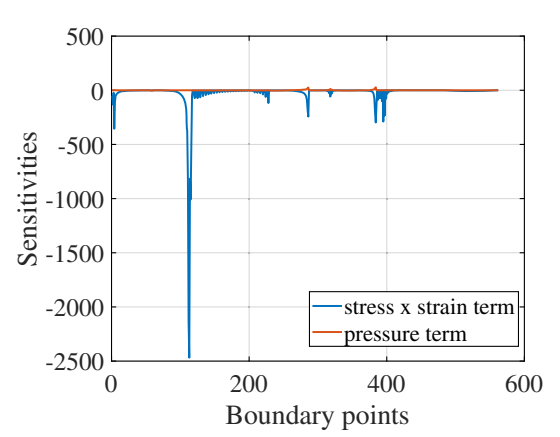

(a)

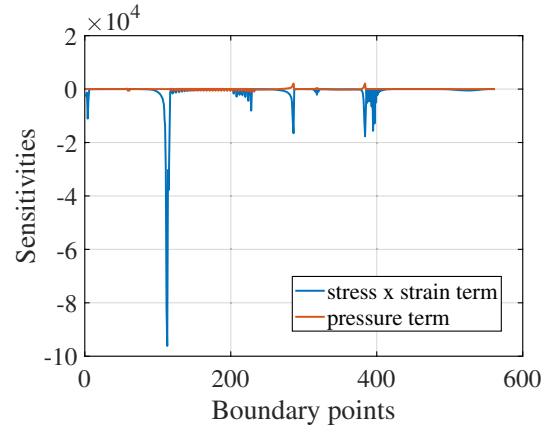

(b) optimization. Also, due to the reinitialization of the level set, tiny holes are formed in the solution as it can be noticed in the converged structural topology. Figure $9 \mathrm{~b}$, however, presents smooth convergence with less structural members breaking during optimization, as indicated by the snapshots of the solution (see Fig. 10).

\subsection{Pressurized chamber}

The pressurized chamber design problem was solved in the pioneering works by Hammer and Olhoff (2000) and Chen and Kikuchi (2001). Besides these two, only Zhang et al. (2008) and Picelli et al. (2015b) solved this problem using the SIMP and the BESO methods, respectively. Therefore, this example is explored less in the literature and to the best of the authors' knowledge, it has not been solved with the LSTO method.

Herein, the schematic problem from Fig. 11 a is solved with $120 \times 76$ quadrilateral elements. The initial fluid region is kept fixed during the optimization and used in the fluid flooding process. Two $40 \times 6$ parts of the structure are considered as non-design domain, as depicted in Fig. 11 a. An inlet pressure $P_{f}=P_{\text {in }}$ is imposed at the right edge of the initial fluid domain and an outlet pressure $P_{\text {out }}=1.0$ per unit length is imposed at the entire bottom edge of the pressurized chamber. The initial solution from Fig. $11 \mathrm{~b}$ is considered for optimization. Problem $P_{2}$ is solved with $\mu=50.0$. Figure 12 presents the final solutions for different values of $P_{0}$.

The chamber topologies from Fig. 12 resemble the ones obtained by the SIMP and the BESO methods (Zhang et al. 2008; Picelli et al. 2015b). One advantage of using the present LSTO technique is to obtain smooth and very well-defined structural boundaries with a relative small grid. For instance, Picelli et al. (2015b) obtained a crisp chamber topology visually similar to the present LSTO solution when using 57000 finite elements, while here we use 9120. Although there is not any other LSTO solution in the literature for this problem, we can point out that the pressure modeling via work equivalent integrals represents a quite simple alternative to other techniques, e.g., XFEM and mixed models. Figure 13 shows the snapshots of the chamber optimization iterations with $P_{0}=10.0$ per unit length, converged to the final compliance of $9.09 \times 10^{5}$ as seen in Fig. 14.

When deriving the compliance function, a term that accounts for the changes in the load due to a change in the design variable should appear in the sensitivity function when considering the design-dependency of the load. For a pressure loading problem, the change in the compliance due to a change in the pressure distribution is represented by the term $2 \operatorname{div}\left(p_{0} u\right)$ in (28) (Allaire et al. 2004).
Fig. 16 Pressurized chamber design via LSTO with a complete sensitivity function evaluation and $\mathbf{b}$ sensitivities computed without the pressure term. Difference in structural compliance of $1.64 \%$

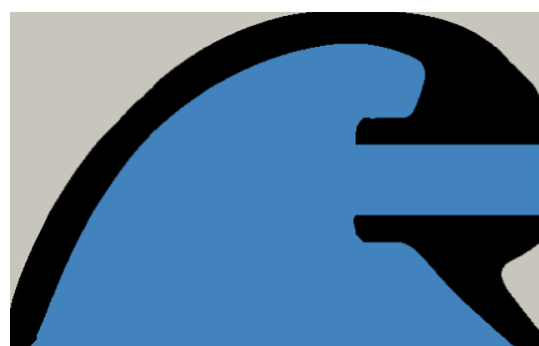

(a) $\mathbf{f}_{p}^{T} \tilde{\mathbf{u}}_{s}=9.09 \times 10^{5}$

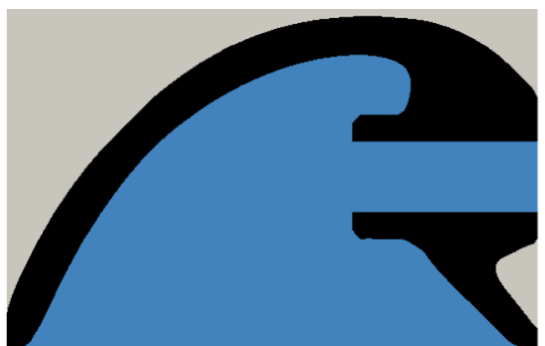

(b) $\mathbf{f}_{p}^{T} \tilde{\mathbf{u}}_{s}=9.24 \times 10^{5}$ 


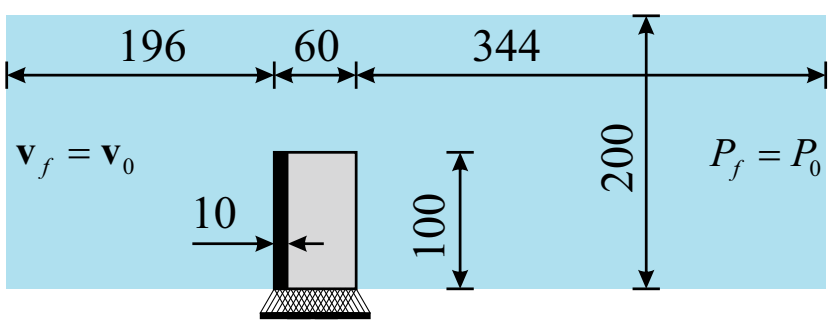

Fig. 17 Beam support design problem

For a full solid pressure chamber, Fig. 15 presents the magnitude of the sensitivity function values for all boundary points separated by its different terms when $P_{\text {in }}=1.0$ and $P_{\text {in }}=10.0$ per unit length. In this case, these values were computed with the sensitivity function discretized by FEM and expressed in (29). Figure 15 shows that the pressure term $2\left(P_{f} \mathbf{B} \tilde{\mathbf{u}}_{s} \cdot\{1,1,0\}+\tilde{\mathbf{u}}_{s} \cdot \nabla \mathbf{N}_{f} P_{f}\right)$ from (29) is generally much lower when compared with the stress $\times$ strain term $\mathbf{D B} \tilde{\mathbf{u}}_{s} \cdot \mathbf{B} \tilde{\mathbf{u}}_{s}$. Pressure $P_{f}$ is only non-zero for boundary points located at the fluid-structure interface and when the pressure values are the same at all points, the gradient term $\nabla \mathbf{N}_{f} P_{f}$ is null. This study shows that the sensitivity of the pressure change in this design optimization problem has a negligible effect. Figure 16 presents the final design solutions when evaluating the completed sensitivity function and when computing sensitivities without the pressure term.

\subsection{Beam support}

The last example considers the beam support design inside a fluid flow channel. The works by Jenkins and Maute (2016), Yoon (2010), Picelli et al. (2017b), and Lundgaard et al. (2018) describe the interaction between the fluid flow governed by the incompressible steady-state Navier-Stokes equations with the linear elastic structures. As proposed by Zhao et al. (2018), an approximate linear model can be used to simplify the flow based on a Darcy potential flow model. Borrowing this idea, herein we rewrite (1) in the form of Poisson's equation as

$\nabla^{2} P_{f}=\mathbf{v}_{f} \quad$ in $\Omega_{f}$,

where $\mathbf{v}_{f}$ is the fluid velocity. The pressure boundary condition is applied as (2) and a velocity profile is imposed as

$\mathbf{v}_{f}=\mathbf{v}_{0}$,

with $\mathbf{v}_{0}$ being the velocity on a portion of the boundary $\Gamma_{f}$.

The design problem in Fig. 17 is considered for optimization. A distributed inlet velocity profile $\mathbf{v}_{0}=1.0$ is applied at the left edge of the fluid domain. An outlet pressure $P_{0}=0.0$ is imposed at the right edge. Problem $P_{2}$ is solved with $\mu=1.0$. The $10 \times 100$ beam in the black region in Fig. 17 is specified as the non-design domain. The mesh of solid finite elements including the design and nondesign domains is $60 \times 100$. For a compliance minimization problem with volume constraint $\bar{V} \leq 30 \%$, Fig. 18 presents the initial and the structural topology solution.

The final solution follows the tendency of obtaining curved structural members similarly to the previous examples of this work. This is expected for a hydrostatic fluid pressure field as arch-like structures are known to bear pressure loads well. The pressure drop in the channel is relatively smooth. If the Navier-Stokes equations were used, the pressure drop would be possibly higher specially for higher Reynolds number, which can significantly change the solution patterns. For future works, this methodology can be extended to different types of fluid-structure interaction.
Fig. 18 Beam support design problem. a Initial guess design and b solution via LSTO

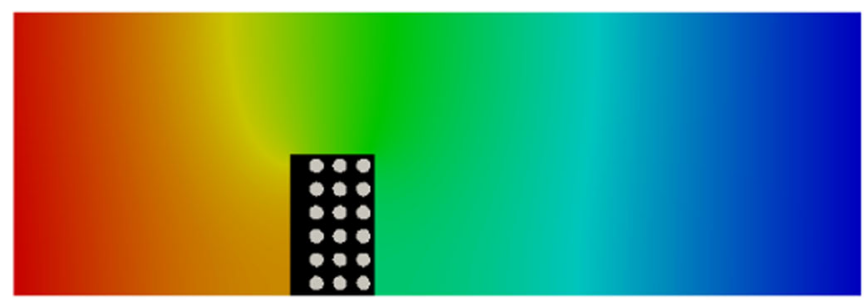

(a)

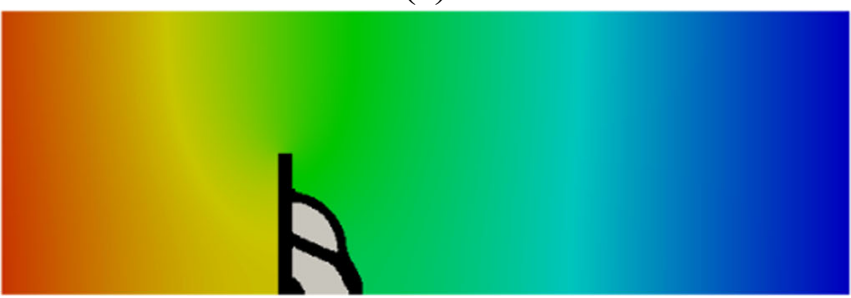

(b)
Pressure

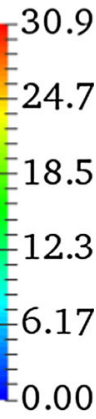




\section{Conclusions}

This paper presented a LSTO method for compliance minimization of structures under design-dependent fluid pressure loads. Laplace's equation was used to solve the fluid pressure field. Using our LSTO method and the classic FE computation of work equivalent loads, pressure loading problems with moving boundaries are addressed. The effects of a perimeter-based regularization were demonstrated. It was shown that smooth boundaries and good convergence are achieved. The topology results agree well with the benchmarking solutions in literature obtained by the other methods available, particularly with SIMP and BESO. The interaction between an elastic structure and fluids happens at the interfaces; therefore, the clear definition of the structural boundaries plays a crucial role in such problems, making the present LSTO method advantageous. Furthermore, the use of classic FE load integration turns the modeling of the pressure surface much simpler than XFEM or mixed models. Another advantage is the application of shape sensitivities. In this context, any fluid-structure discretization can be used as the sensitivities are in the continuum space. The method herein can be used as starting point for more complex design-dependent physics problems.

\section{Replication of results}

The data from these numerical investigations are available by contacting the authors.

Acknowledgments The authors would like to thank the Numerical Analysis Group at the Rutherford Appleton Laboratory for their FORTRAN HSL packages (HSL, a collection of Fortran codes for large-scale scientific computation. See http://www.hsl.rl.ac.uk/).

Funding information This study was supported by the São Paulo Research Foundation (FAPESP), grants 2018/05797-8 and 2019/01685-3, and the Engineering and Physical Sciences Research Council (EPSRC), fellowship grant EP/M002322/2.

\section{Compliance with ethical standards}

Conflict of interest The authors declare that they have no conflict of interest.

Open Access This article is distributed under the terms of the Creative Commons Attribution 4.0 International License (http:// creativecommons.org/licenses/by/4.0/), which permits unrestricted use, distribution, and reproduction in any medium, provided you give appropriate credit to the original author(s) and the source, provide a link to the Creative Commons license, and indicate if changes were made.

\section{References}

Alexandersen J, Sigmund O, Aage N (2016) Large scale threedimensional topology optimisation of heat sinks cooled by natural convection. Int J Heat Mass Transf 100:876-891

Allaire G, Jouve F, Toader AM (2004) Structural optimization using sensitivity analysis and a level-set method. J Comput Phys 194:363-393

Azevedo FM, Moura MS, Vicente WM, Picelli R, Pavanello R (2018) Topology optimization of reactive acoustic mufflers using a bidirectional evolutionary optimization method. Struct Multidiscip Optim 58(5):2239-2252

Bathe KJ (2006) Finite element procedures. Prentice Hall, Englewood Cliffs

Bendsøe M (1995) Optimization of structural topology, shape, and material. Springer, Berlin

Bourdin B, Chambolle A (2003) Design-dependent loads in topology optimization. ESAIM Control Optim Calc Var 9:19-48

Brezzi F, Fortin M (1991) Mixed and hybrid finite element methods. Springer, Berlin

Chakraborty S, Goswami S, Rabczuk T (2019) A surrogate assisted adaptive framework for robust topology optimization. Comput Methods Appl Mech Eng 346:63-84

Chen BC, Kikuchi N (2001) Topology optimization with designdependent loads. Finite Elem Anal Des 37:57-70

Cook RD, Malkus DS, Plesha ME, Witt RJ (2002) Concepts and applications of finite element analysis, 4th edn. Wiley, New York

Deaton JD, Grandhi RV (2014) A survey of structural and multidisciplinary continuum topology optimization: post 2000 . Struct Multidiscip Optim 49:1-38

Deaton JD, Grandhi RV (2016) Stress-based design of thermal structures via topology optimization. Struct Multidiscip Optim 53:253-270

Du J, Olhoff N (2004) Topological optimization of continuum structures with design-dependent surface loading. Part I: new computational approach for 2D problems. Struct Multidiscip Optim 27:151-165

Dunning PD, Ovtchinnikov E, Scott J, Kim HA (2016) Level-set topology optimization with many linear buckling constraints using an efficient and robust eigensolver. Int $\mathrm{J}$ Numer Methods Eng 1029-1053(12):1029-1053

Emmendoerfer H, Fancello EA, Silva ECN (2018) Level set topology optimization for design-dependent pressure load problems. Int $\mathbf{J}$ Numer Methods Eng 115(7):825-848

Guest JK, Prévost JH (2006) Optimizing multifunctional materials: design of microstructures for maximized stiffness and fluid permeability. Int J Solids Struct 43(22-23):70287047

Hammer VB, Olhoff N (2000) Topology optimization of continuum structures subjected to pressure loading. Struct Multidiscip Optim 19:85-92

Hedges LO, Kim HA, Jack RL (2017) Stochastic level-set method for shape optimisation. J Comput Phys 348:82-107

Huang X, Xie YM (2011) Evolutionary topology optimization of continuum structures including design-dependent self-weight loads. Finite Elem Anal Des 47:942-948

Isakari H, Kondo T, Takahashi T, Matsumoto T (2017) A level-setbased topology optimisation for acoustic-elastic coupled problems with a fast BEM-FEM solver. Comput Methods Appl Mech Eng 315:501-521

Jenkins N, Maute K (2016) An immersed boundary approach for shape and topology optimization of stationary fluid-structure interaction problems. Struct Multidiscip Optim 52(1):179-195 
Lee E, Martins JRRA (2012) Structural topology optimization with design-dependent pressure loads. Comput Methods Appl Mech Eng 233-236:40-48

Lundgaard C, Alexandersen J, Zhou M, Andreasen C, Sigmund O (2018) Revisiting density-based topology optimization for fluid-structure-interaction problems. Struct Multidiscip Optim 58(3):969-995

Noguchi Y, Yamamoto T, Yamada T, Izui K (2017) A level set-based topology optimization method for simultaneous design of elastic structure and coupled acoustic cavity using a two-phase material model. J Sound Vib 404:15-30

Osher S, Fedkiw R (2003) Level set methods and dynamic implicit surfaces. Applied mathematical sciences, vol 153. Springer, New York

Picelli R, Vicente WM, Pavanello R (2015a) Bi-directional evolutionary structural optimization for design-dependent fluid pressure loading problems. Eng Optim 47(10):1324-1342

Picelli R, Vicente WM, Pavanello R, Xie YM (2015b) Evolutionary topology optimization for natural frequency maximization problems considering acoustic-structure interaction. Finite Elem Anal Des 106:56-64

Picelli R, van Dijk R, Vicente WM, Pavanello R, Langelaar M, van Keulen F (2017a) Topology optimization for submerged buoyant structures. Eng Optim 49(1):1-21

Picelli R, Vicente WM, Pavanello R (2017b) Evolutionary topology optimization for structural compliance minimization considering design-dependent FSI loads. Finite Elem Anal Des 135:44-55

Picelli R, Townsend S, Brampton C, Norato J, Kim HA (2018a) Stressbased shape and topology optimization with the level set method. Comput Methods Appl Mech Eng 329:1-23

Picelli R, Townsend S, Kim HA (2018b) Stress and strain control via level set topology optimization. Struct Multidiscip Optim 58:2037-2051

Sethian JA (1996) A fast marching level set method for monotonically advancing fronts. Proc Natl Acad Sci 93(4):1591-1595

Shu L, Wang MY, Ma Z (2014) Level set based topology optimization of vibrating structures for coupled acoustic-structural dynamics. Comput Struct 132:34-42

Sigmund O (2007) Morphology-based black and white filters for topology optimization. Struct Multidiscip Optim 33(4-5):401-424

Sigmund O, Clausen PM (2007) Topology optimization using a mixed formulation: an alternative way to solve pressure load problems. Comput Methods Appl Mech Eng 196:1874-1889

Sivapuram R, Picelli R (2018) Topology optimization of binary structures using integer linear programming. Finite Elem Anal Des 139:49-61

Sutradhar A, Paulino GH, Miller MJ, Nguyen TH (2010) Topological optimization for designing patient-specific large craniofacial segmental bone replacements. Proc Natl Acad Sci USA 107(30):13222-13227

Townsend S, Picelli R, Stanford B, Kim HA (2018) Structural optimization of plate-like aircraft wings under flutter and divergence constraints. AIAA J 56(8):3307-3319

Vicente WM, Picelli R, Pavanello R, Xie YM (2015) Topology optimization of frequency responses of fluid-structure interaction systems. Finite Elem Anal Des 98:1-13

Wang C, Zhao M, Ge T (2016) Structural topology optimization with design-dependent pressure loads. Struct Multidiscip Optim 53:1005-1018

Xia Q, Wang MY, Shi T (2015) Topology optimization with pressure load through a level set method. Comput Methods Appl Mech Eng 283:177-195

Yoon GH (2010) Topology optimization for stationary fluid-structure interaction problems using a new monolithic formulation. Int $\mathrm{J}$ Numer Methods Eng 82:591-616

Yoon GH, Jensen JS, Sigmund O (2007) Topology optimization of acoustic-structure problems using a mixed finite element formulation. Int J Numer Methods Eng 70:1049-1075

Zhang H, Zhang X, Liu S (2008) A new boundary search scheme for topology optimization of continuum structures with designdependent loads. Struct Multidiscip Optim 37:121-129

Zhao X, Zhou M, Sigmund O, Andreasen CS (2018) A “poor man's approach" to topology optimization of cooling channels based on a Darcy flow model. Int J Heat Mass Transf 116:1108-1123

Zienkiewicz OC, Taylor RL (2005) The finite element method, vol 1-3, 6th edn. Elsevier Butterworth Heinemann, Oxford

Publisher's note Springer Nature remains neutral with regard to jurisdictional claims in published maps and institutional affiliations. 